\title{
AVALIAÇÃO DA VIABILIDADE TÉCNICA E ECONÔMICA DA REUTILIZAÇÃO DA ÁGUA DE RINSAGEM DE EMBALAGENS DESCARTÁVEIS
}

\author{
Ismael Magagnin ${ }^{1}$, Gustavo Reisdörfer ${ }^{2}$
}

\begin{abstract}
Resumo: A humanidade entende que a água é imprescindível para sua vida, assim como para o desenvolvimento de sua coletividade, sendo utilizada em diversas atividades do cotidiano, inclusive em indústrias, as quais correspondem a segunda maior demanda do seu consumo, ficando atrás apenas da demanda utilizada na agricultura. Tendo em vista que o conceito de reuso da água não é algo novo, e se faz necessário, o presente trabalho tem por finalidade avaliar a possibilidade de reuso da água do processo de rinsagem de embalagens descartáveis, do processo produtivo de uma indústria de refrigerantes. Primeiramente foram avaliadas as características físicas, químicas e microbiológicas da água de abastecimento e descarte do rinser, e após da água de reciclo. Foram realizados testes em escala piloto, com volume de até 72.000 unidades. Os resultados apresentaram-se satisfatórios, possibilitando a aplicação do sistema de reciclo da água de rinsagem, com uma redução aproxima de $97 \%$ do consumo de água e $78 \%$ do custo financeiro do processo, em comparação ao sistema utilizado atualmente.
\end{abstract}

Palavras-chave: Água. Reuso. Tratamento.

Abstract: Humanity understands that water is essential for its life, as well as for the development of its collectivity, being used in various activities of daily life, including in industries, which correspond to the second greater demand of its consumption, being behind only the used demand in the farming. Considering that the concept of water reuse is not something new, and it is necessary, the present work has the purpose of evaluating the possibility of reuse of water from the process of disposable packaging, the production process of a soft drink industry. First, the physical, chemical and microbiological characteristics of the water supply and disposal of the rinser and after the recycle water were evaluated. Pilot scale tests were performed, with a volume of up to 72,000 units. The results were satisfactory, allowing the application of the recycling system of the rinsing water, with a reduction of approximately $97 \%$ of water consumption and $78 \%$ of the financial cost of the process, compared to the system currently used.

Keywords: Water. Reuse. Treatment.

1 Graduando do Curso de Engenharia de Alimentos - Centro Universitário UNIVATES - Lajeado RS - Brasil. ismaelm@universo.univates.br

2 Professor Mestre da Universidade do Vale do Taquari - Univates - Lajeado - RS - Brasil. Orientador do trabalho de Ismael Magagnin. greisdorfer@univates.br 


\section{INTRODUÇÃO}

A humanidade reconhece a água como algo indispensável para a manutenção da vida, assim como sua utilização para a evolução da sua raça. (HELLER, 2006; LIU et al., 2010). A água é utilizada em diversas atividades para manutenção do cotidiano humano, assim como em processos industriais, onde atua como matéria-prima, veículo, refrigeração, procedimentos de higienização, dentre outras necessidades do setor (MIERZWA \& HESPANHOL, 2005; RIBEIRO, 2008; ANA, 2016). Representa um dos principais recursos necessários nas indústrias, no segmento de bebidas, onde se utiliza entre 1,63 e 13,0 $\mathrm{m}^{3}$ de água para cada $1,0 \mathrm{~m}^{3}$ de bebida produzida, considerando todas as etapas necessárias para o processamento dos produtos. (SRHU/MMA, 2009).

O conceito de reuso de água não é novo, sendo relatado já na Grécia Antiga. Atualmente há uma constante abordagem deste assunto através de políticas governamentais e não governamentais para a racionalização do uso deste recurso (RODRIGUES, 2005). O uso de água de reuso para fins potáveis deve atender a Portaria MS no 2.914, de 12 de dezembro de 2011, que dispõe sobre os procedimentos de controle e de vigilância da qualidade da água para consumo humano e seu padrão de potabilidade. Para o tratamento ser considerado satisfatório este deverá apresentar eficiência, operação fácil e econômica, além de atender as características físicas, químicas e biológicas da legislação correspondente.

O objetivo deste trabalho foi avaliar a viabilidade técnica e econômica de tratamento e reuso da água do processo de rinsagem de embalagens descartáveis em uma indústria de refrigerantes. A rinsagem é uma etapa do processo produtivo da indústria de bebidas não alcoólicas, na qual as embalagens descartáveis são submetidas à desinfecção, através de uma substância com eficiência comprovada, antes do envase. As embalagens são suspensas com o gargalo para baixo e, a rinsagem é realizada através de um jato de água com pressão e concentração de desinfetante (controlada), a fim de atender o objetivo desta etapa.

\section{METODOLOGIA}

\subsection{Quantificação do volume de água gerado ao tratamento de efluentes no processo de rinsagem}

Primeiramente, foi instalado um hidrômetro com as características exigidas pela ABNT e INMETRO na entrada de abastecimento da água do rinser. Durante cinco dias de produção, foi controlada a vazão de água utilizada durante o processo produtivo de rinsagem, através da leitura do hidrômetro ao final deste período. A partir desta leitura foi calculado o consumo de água por unidade, conforme Equação 1:

Equação 1: Consumo de água/unidade = (leitura final-leitura inicial)/número de unidades rinsadas 
Através da verificação do volume de água necessário para rinsagem de uma embalagem foi calculado o volume de água utilizado no ano de 2016 (doze meses de produção), considerando como base de cálculo a Equação 2:

Equação 2: volume de água necessária/ano = consumo de água/unidade $\times$ número de unidades/ano

Através deste cálculo foi possível identificar o volume de água necessário para a produção dos últimos doze meses, o qual foi utilizado para realização do estudo de viabilidade econômica. O volume de água necessário para o abastecimento desta etapa é considerado o mesmo que é descartado para o tratamento de efluentes.

Para realização do estudo de viabilidade econômica, através da comparação entre os sistemas, considerou-se o volume total produzido na linha de produção do ano de 2016.

\subsection{Caracterização da água de processo}

As amostras de água de abastecimento e saída do rinser foram coletadas de acordo com as recomendações descritas no livro Standard Methods for the Examination of Waterand Wasterwater. A água utilizada é captada de poços artesianos, filtrada e desinfetada com hipoclorito de sódio. As coletas foram realizadas durante cinco dias.

As análises físicas, químicas e microbiológicas realizadas estão descritas na Tabela 1, em conformidade com a Portaria MS no 2.914/2011.

Tabela 1 Análises selecionadas para caracterização das águas

\begin{tabular}{l|l}
\hline Físicas e químicas & Microbiológica \\
\hline Cloro residual livre & Coliformes totais \\
\hline Condutividade & Escherichia coli \\
\hline $\mathrm{pH}$ & Bactérias totais \\
\hline Turbidez & Bolores e leveduras \\
\hline
\end{tabular}

Fonte: Portaria MS no 2.914/2011.

A Figura 1 apresenta os pontos de coleta das amostras, assim como uma representação geral do sistema, objeto deste estudo. 
Figura 1 - Identificação dos pontos de coletas

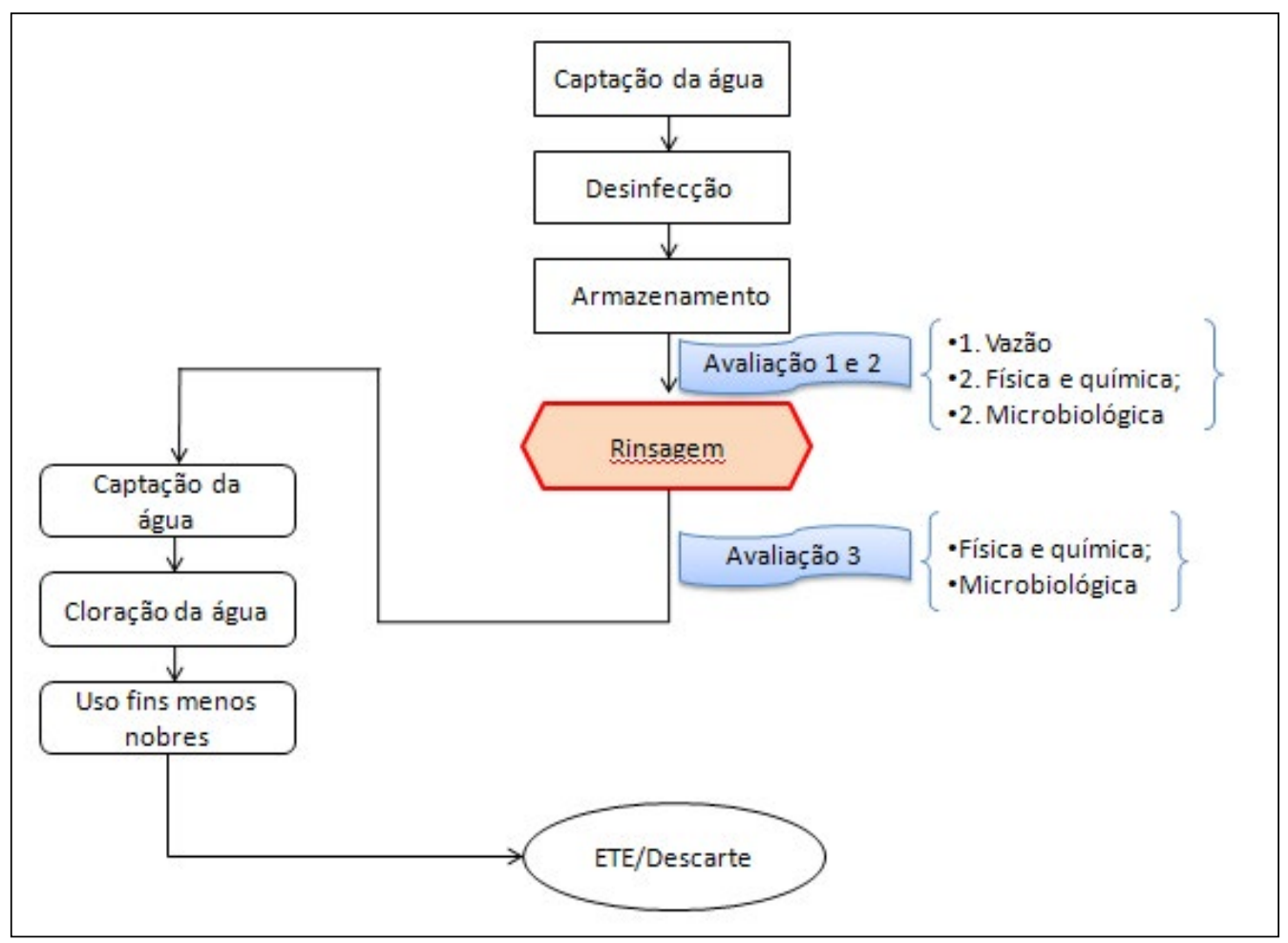

Fonte: Dos autores.

\subsection{Definição do tratamento utilizado}

Os resultados encontrados através das análises das águas coletadas foram avaliados quanto aos parâmetros físicos, químicos e microbiológicos. Esta caracterização foi necessária para definir o método de tratamento mais adequado para o reciclo, tanto do ponto de vista técnico quanto econômico.

\subsection{Avaliação física, química e microbiológica da água de reciclo}

Nesta fase foram iniciadas as análises físicas, químicas e microbiológicas da água de reciclo utilizada no rinser, bem como seu tratamento, o qual consistiu em introdução de hipoclorito de sódio, seguido por filtração. Os testes foram realizados em escala gradativa, iniciando-se com 1.000 unidades, após 10.000 unidades e na etapa final, com 72.000 unidades, volume referente a um dia de produção. 
Os parâmetros físicos, químicos e microbiológicos utilizados, seguiram os padrões descritos na Portaria MS no 2.914/2011 e, na sua ausência foram considerados os padrões definidos pela empresa, conforme a Tabela 2:

Tabela 2 - Padrões físicos, químicos e microbiológicos para água de reciclo

\begin{tabular}{|c|c|c|}
\hline Parâmetro & Limite & Referência \\
\hline Coliformes termotolerantes & Ausência em 100 mL & \begin{tabular}{|l|} 
Portaria MS no \\
$2.914 / 2011$ \\
\end{tabular} \\
\hline Escherichia coli & Ausência em 100 mL & $\begin{array}{l}\text { Portaria MS no } \\
2.914 / 2011 \\
\end{array}$ \\
\hline Bolores & Máximo $10 \mathrm{UFC} / \mathrm{mL}$ & Padrão interno empresa \\
\hline Leveduras & Máximo 10 UFC/mL & Padrão interno empresa \\
\hline Bactérias totais & Máximo 70 UFC/100 mL & Padrão interno empresa \\
\hline Cloro residual livre & $\begin{array}{l}\text { Mínimo 0,5 mg/L } \\
\text { Máximo } 10 \mathrm{mg} / \mathrm{L}\end{array}$ & Padrão interno empresa \\
\hline Turbidez & Máximo 5 uT & $\begin{array}{l}\text { Portaria MS no } \\
2.914 / 2011 \\
\end{array}$ \\
\hline Condutividade & Máximo $250 \mu \mathrm{S} / \mathrm{cm}^{2}$ & Padrão interno empresa \\
\hline $\mathrm{pH}$ & $\begin{array}{l}\text { Mínimo 6,5 } \\
\text { Máximo 7,7 } \\
\end{array}$ & Padrão interno empresa \\
\hline Alcalinidade & $\begin{array}{l}\text { Mínimo } 25 \mathrm{mg} \mathrm{CaCO} \\
\text { Máximo } 55 \mathrm{mg} \mathrm{CaCO}\end{array}$ & Padrão interno empresa \\
\hline
\end{tabular}

Fonte: Portaria MS no 2.914/2011 e Padrão Interno empresa.

A Figura 2 apresenta o ponto de coleta da água de reciclo, assim como o sistema geral de reciclo da água para rinsagem. 
Figura 2 - Identificação do sistema de reciclo proposto

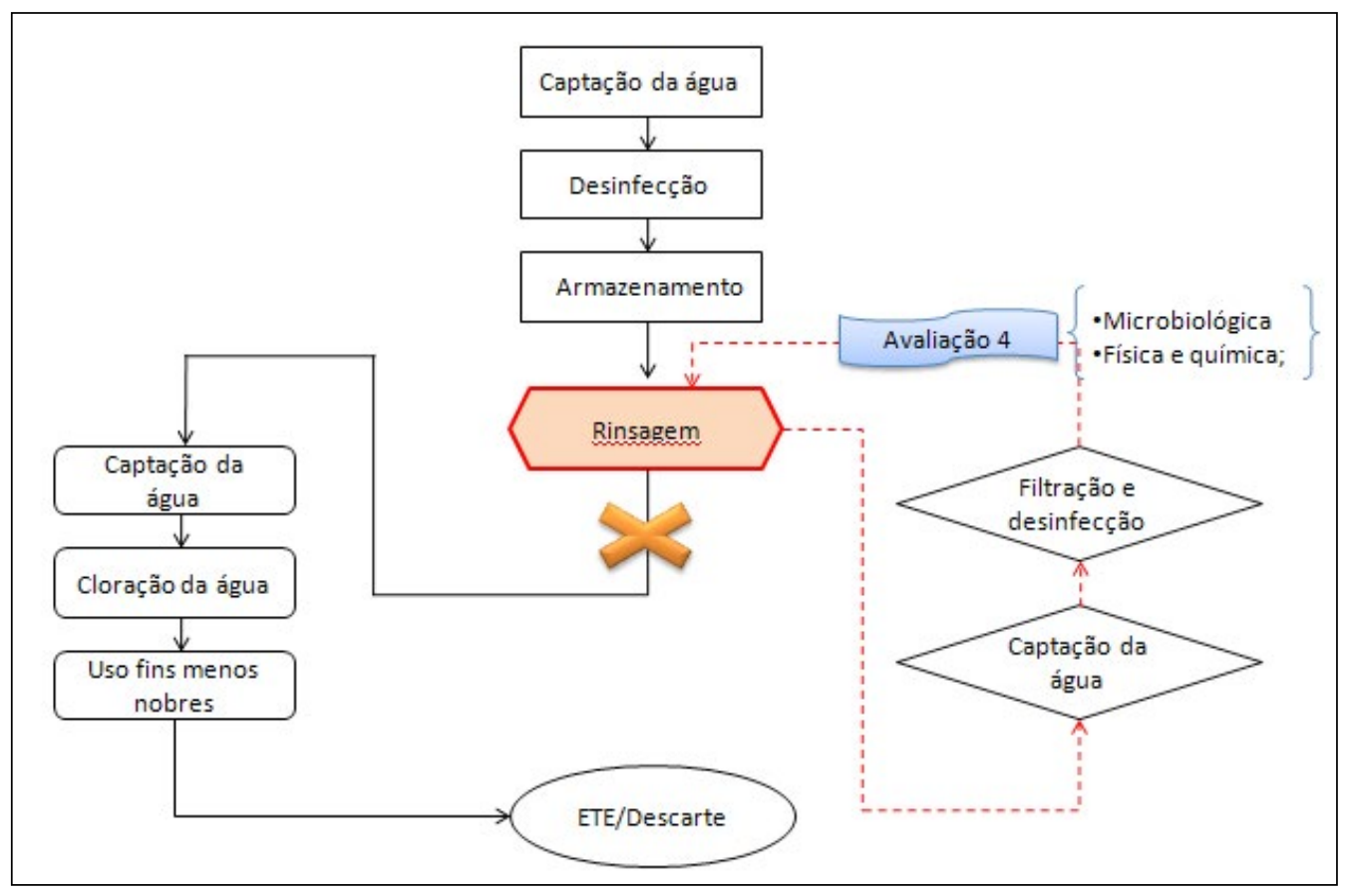

Fonte: Dos autores.

\subsection{Avaliação econômica da operação dos sistemas de rinsagem}

Este estudo seguiu os princípios básicos do programa Produção Mais Limpa, ferramenta que vem sendo utilizada pelas indústrias e apresentando resultados satisfatórios quanto aos aspectos social e econômico.

Para realização desta avaliação foram considerados os custos relacionados ao processo de rinsagem: tratamento de efluentes, manutenção preventiva, captação de água e filtros, através da Equação 3:

Equação 3: Custo convencional - Custo do projeto proposto = Economia estimada 


\section{RESULTADOS E DISCUSSÃO}

\subsection{Quantificação do volume de água gerado ao tratamento de efluentes no processo de rinsagem}

O volume obtido da medição através do hidrômetro foi de $250 \mathrm{~mL}$ de água por unidade produzida no sistema utilizado atualmente (volume correspondente ao gerado ao tratamento de efluentes). Considerando a produção total do ano de 2016, o volume necessário ao processo de rinsagem correspondeu a 10.508.273,25 de litros de água. Já no sistema proposto, onde a água foi utilizada por diversos ciclos, durante um dia de produção (24 horas), o volume necessário em um ano corresponderia a 264.689,44 litros, gerando uma redução superior a $97 \%$ de litros de água, ou seja, esta economia seria suficiente para abastecer 140 pessoas durante um ano, de acordo com a Companhia de Saneamento Básico do Estado de São Paulo (SABESP) que estima que consumo médio de água do brasileiro seja de 200 litros/pessoa/dia.

\subsection{Caracterização da água de processo}

As médias dos resultados das análises físicas e químicas e das análises microbiológicas das cinco coletas realizadas, seguem descritos nas Tabelas 3 e 4, respectivamente:

Tabela 3 - Caracterização física e química da água

\begin{tabular}{c|c|c|c|c|c}
\hline Local & $\begin{array}{c}\text { Cloro livre } \\
(\mathrm{mg} / \mathrm{L})\end{array}$ & $\begin{array}{c}\text { Condutividade } \\
\left(\mu \mathrm{S} / \mathrm{cm}^{2}\right)\end{array}$ & $\mathrm{pH}$ & $\begin{array}{c}\text { Turbidez } \\
(\mathrm{uT})\end{array}$ & $\begin{array}{c}\text { Alcalinidade } \\
\left(\mathrm{mg} \mathrm{CaCO}_{3}\right)\end{array}$ \\
\hline Água abastecimento & $0,73 \pm 0,21$ & $184 \pm 17,26$ & $7,53 \pm 0,23$ & $0,45 \pm 0,22$ & $44,9 \pm 1,54$ \\
\hline Água saída do rinser & $0,65 \pm 0,90$ & $181 \pm 17,29$ & $7,39 \pm 0,19$ & $0,63 \pm 0,38$ & $36,6 \pm 2,58$ \\
\hline
\end{tabular}

Fonte: Dos autores.

Tabela 4 - Caracterização microbiológica da água

\begin{tabular}{c|c|c|c|c|c}
\hline Local & $\begin{array}{c}\text { Bolor } \\
(\mathrm{UFC} / 100 \\
\mathrm{mL})\end{array}$ & $\begin{array}{c}\text { Leveduras } \\
(\mathrm{UFC} / 100 \\
\mathrm{mL})\end{array}$ & $\begin{array}{c}\text { Coliformes } \\
\text { termotolerantes } \\
(\mathrm{UFC} / 100 \mathrm{~mL})\end{array}$ & $\begin{array}{c}\text { Escherichia coli } \\
(\mathrm{UFC} / 100 \mathrm{~mL})\end{array}$ & $\begin{array}{c}\text { Bactérias totais } \\
(\mathrm{UFC} / 100 \mathrm{~mL})\end{array}$ \\
\hline $\begin{array}{c}\text { Água de } \\
\text { abastecimento }\end{array}$ & $3 \pm 1,12$ & $0 \pm 0$ & $0 \pm 0$ & $0 \pm 0$ & $19 \pm 42,27$ \\
\hline $\begin{array}{c}\text { Água saída do } \\
\text { rinser }\end{array}$ & $9 \pm 1,49$ & $0 \pm 0$ & $0 \pm 0$ & $0 \pm 0$ & $50 \pm 67,55$ \\
\hline
\end{tabular}

Fonte: Dos autores. 
Os resultados encontrados na água de abastecimento apresentam-se dentro dos padrões internos da empresa, bem como os padrões descritos na legislação vigente (Portaria MS no 2.914/2011). A água de saída do rinser apresentou ligeira acidificação, no entanto, permanecendo dentro dos padrões exigidos, sendo estes os mesmos para a água de abastecimento. Os resultados microbiológicos apresentarem-se dentro dos padrões legais, sendo que não variaram para leveduras, coliformes termotolerantes e Escherichia coli. Com relação a bolores e bactérias totais, as análises da água de saída do rinser, apresentaram valores maiores que os encontrados na água de abastecimento, ainda assim mantiveram-se em conformidade com os padrões descritos na Tabela 2.

\subsection{Definição do tratamento utilizado}

Apesar da ligeira diferença encontrada nas análises físicas, químicas e microbiológicas na água de saída do rinser, em relação à água de abastecimento, optouse por utilizar o sistema de tratamento utilizado atualmente, devido à facilidade de operação e o baixo custo envolvido, o qual consiste em filtração e desinfecção. De acordo com Richter, 1991, o tratamento da água escolhido, deve utilizar apenas os processos necessários para almejar a qualidade ideal, com baixo custo de operação, atendendo a legislação vigente.

\subsection{Avaliação física, química e microbiológica da água de reciclo}

Conforme exposto na Tabela 5, as análises físicas e químicas apresentaram considerável alteração de turbidez, devido à presença de algumas substâncias insolúveis na água, observadas durante as coletas, ainda assim, em concordância com os padrões descritos na Tabela 2. Os parâmetros de condutividade, $\mathrm{pH}$ e alcalinidade apresentaram tendência à redução, mantendo-se entre os padrões estabelecidos, não representando comprometimento do controle do processo.

Tabela 5 - Avaliação físico-química da água de reciclo

\begin{tabular}{c|c|c|c|c|c}
\hline Unidades & $\begin{array}{c}\text { Cloro livre } \\
(\mathrm{mg} / \mathrm{L})\end{array}$ & $\begin{array}{c}\text { Condutividade } \\
\left(\mu \mathrm{S} / \mathrm{cm}^{2}\right)\end{array}$ & $\mathrm{pH}$ & $\begin{array}{c}\text { Turbidez } \\
(\mathrm{uT})\end{array}$ & $\begin{array}{c}\text { Alcalinidade } \\
\left(\mathrm{mg} \mathrm{CaCO}_{3}\right)\end{array}$ \\
\hline 0 & 10,3 & 202 & 7,68 & 0,5 & 51,9 \\
\hline 1.000 & 9,1 & 173 & 7,49 & 0,72 & 48,6 \\
\hline 10.000 & 8,7 & 165,3 & 7,31 & 1,3 & 46,6 \\
\hline 72.000 & 8,2 & 166,2 & 7,27 & 0,49 & 46,3 \\
\hline
\end{tabular}

Fonte: Dos autores.

Na Tabela 6 são apresentados os resultados das análises microbiológicas: 
Tabela 6 - Avaliação microbiológica da água de reciclo

\begin{tabular}{l|c|c|c|c|c}
\hline \multicolumn{1}{c|}{ Coleta } & $\begin{array}{c}\text { Bolor } \\
(\mathrm{UFC} / 100 \mathrm{~mL})\end{array}$ & $\begin{array}{c}\text { Leveduras } \\
(\mathrm{UFC} / 100 \mathrm{~mL})\end{array}$ & $\begin{array}{c}\text { Coliformes } \\
\text { termotolerantes } \\
(\mathrm{UFC} / 100 \mathrm{~mL})\end{array}$ & $\begin{array}{c}\text { Escherichia coli } \\
(\mathrm{UFC} / 100 \mathrm{~mL})\end{array}$ & $\begin{array}{c}\text { Bactérias totais } \\
(\mathrm{UFC} / 100 \mathrm{~mL})\end{array}$ \\
\hline $\begin{array}{l}\text { Água de } \\
\text { abastecimento }\end{array}$ & 3 & 0 & 0 & 0 & 0 \\
\hline $\begin{array}{l}\text { Água após } \\
\text { enxágue de } \\
1.000 \text { unidades }\end{array}$ & 8 & 5 & 0 & 0 & 10 \\
\hline $\begin{array}{l}\text { Água após } \\
\text { enxágue } \\
\text { de } 10.000\end{array}$ & 4 & 0 & 0 & 0 & 0 \\
unidades & & 0 & 0 & 0 & 0 \\
\hline $\begin{array}{l}\text { Água após } \\
\text { enxágue } \\
\text { de 72.000 } \\
\text { unidades }\end{array}$ & 5 & 0 & & & 0 \\
\hline
\end{tabular}

Fonte: Dos autores.

De acordo com a Tabela 6, é possível verificar que não houve diferença nos resultados das análises de coliformes termotolerantes e Escherichia coli na água de enxágue após 1.000, 10.000 e 72.000 unidades, em relação à água de abastecimento no rinser. Os valores encontrados para bactérias totais estão entre os padrões citados na Tabela 2 , sem variação significativa.

Segundo Morais et.al., 2003, Agência Nacional de Vigilância Sanitária (ANVISA) adotou a Resolução no 12/2001, que dispõe sobre o Regulamento Técnico que define os padrões microbiológicos para alimentos, a qual revogou a contagem de bolores e leveduras das análises de refrigerantes, transferindo o monitoramento destes parâmetros às indústrias. Sendo assim, a indústria onde este estudo foi realizado optou por manter os padrões anteriormente exigidos por este órgão, por cautela e interesse em conservar a qualidade microbiológica dos produtos. Os resultados encontrados para bolores e leveduras, apresentam-se entre estes parâmetros.

De acordo com a Instrução Normativa (IN) no 5, 2000, do Ministério da Agricultura, Pecuária e Abastecimento (MAPA) que aprova o Regulamento Técnico para a fabricação de bebidas e vinagres, inclusive vinhos e derivados da uva e do vinho, dirigido a estabelecimentos elaboradores e ou industrializadores, a água de reciclo deve ser mantida sob monitoramento contínuo, a fim de evitar contaminações capazes de comprometer a qualidade do produto final, trazendo riscos à saúde do consumidor.

É importante salientar que os valores das contagens microbiológicas obtidos nas análises da água de enxágue do rinser não divergiram significativamente dos valores 
encontrados na água de abastecimento do rinser, o que representa fator positivo para implantação deste projeto em escala industrial.

\subsection{Avaliação econômica da operação dos sistemas de rinsagem}

$\mathrm{Na}$ tabela 7 são apresentados os principais custos de operação do sistema atual, comparados ao sistema proposto:

Tabela 7 - Avaliação financeira do comparativo atual com o proposto

\begin{tabular}{l|r|r|r}
\hline Descrição & \multicolumn{1}{|l|}{ Sistema atual } & \multicolumn{1}{|c|}{ Sistema proposto } & Economia estimada \\
\hline Tratamento de efluentes & $\mathrm{R} \$ 15.026,83$ & $\mathrm{R} \$ 343,20$ & $\mathrm{R} \$ 14.683,63$ \\
\hline Manutenção preventiva & $\mathrm{R} \$ 2.455,45$ & $\mathrm{R} \$ 2.455,45$ & $\mathrm{R} \$-$ \\
\hline Captação & $\mathrm{R} \$ 8.406,62$ & $\mathrm{R} \$ 211,20$ & $\mathrm{R} \$ 8.195,42$ \\
\hline Filtro & $\mathrm{R} \$ 3.172,80$ & $\mathrm{R} \$ 3.172,80$ & $\mathrm{R} \$-$ \\
\hline Total & $\mathbf{R} \$ \mathbf{2 9 . 0 6 1 , 7 0}$ & $\mathbf{R} \mathbf{6 . 1 8 2 , 6 5}$ & $\mathbf{R} \mathbf{2 2 . 8 7 9 , 0 5}$ \\
\hline
\end{tabular}

Fonte: Dos autores.

Considerando os valores expostos na Tabela 7, é possível identificar decréscimo considerável dos custos com este processo, o qual representa uma redução de aproximadamente $78 \%(\mathrm{R} \$ 22.879,05)$, utilizando-se o sistema objeto deste trabalho em relação ao sistema atualmente utilizado. O valor encontrado representa a economia financeira anual, considerando-se como base o volume produzido no ano de 2016.

É possível visualizar um maior impacto financeiro nos custos designados ao tratamento de efluentes, visto que o volume de descarte da água de rinsagem seria reduzido significativamente. Como consequência do reciclo da água de rinsagem, o volume de captação de água também seria reduzido expressivamente.

\section{CONCLUSÃO}

Os resultados das análises deste estudo mantiveram-se entre os parâmetros estabelecidos nas legislações vigentes, assim como com os parâmetros internos da empresa, garantindo a qualidade do produto final.

A principal barreira encontrada para implementação desta proposta está relacionada ao aspecto cultural, visto que os sistemas de rinsagem atuais normalmente utilizam água de primeiro uso, sem reciclo.

A avaliação realizada corresponde a uma pequena amostragem, necessitando testes adicionais para monitoramento do processo, como por exemplo, a implantação de testes 
microbiológicos de resposta mais rápida, visto que os atuais necessitam de três a cinco dias, assim podendo prolongar o tempo de reciclo para um maior número de unidades e, portanto, produzir conclusões mais assertivas.

Conclui-se que processo de reciclo da água do rinser demonstra potencial para aplicação, dos pontos de vista técnico e econômico, visto que proporcionaria uma economia de aproximadamente $853,69 \mathrm{~m}^{3}$ de água mensais na linha de produção avaliada. Este valor representa não apenas uma economia financeira, mas também elimina uma necessidade de extração da fonte, diminuindo gradativamente sua vazão e consequentemente aumentando sua vida útil.

Tendo em vista que este estudo foi realizado em escala piloto, em uma linha de produção e que esta unidade fabril conta com outras seis linhas, há a possibilidade de expansão, chegando a números mais significativos.

\section{REFERENCIAS}

ABIR, Associação Brasileira das Indústrias de Refrigerantes e de Bebidas não Alcoólicas, 2016. Disponível em: <https://abir.org.br>. Acessado em 02 de setembro de 2016.

ANA, Agência Nacional de Águas, 2013, Disponível em: <http://arquivos.ana.gov.br/ institucional/spr/conjuntura/ANA_Conjuntura_Recursos_Hidricos_Brasil/ANA_

Conjuntura_Recursos_Hidricos_Brasil_2013_Final.pdf>. Acessado em 08 de agosto de 2016.

ANVISA, Agência Nacional de Vigilância Sanitária, 2011. Disponível em: <http://www. saude.mg.gov.br/images/documentos/PORTARIA\%20No-\%202.914,\%20DE\%2012\%20

DE\%20DEZEMBRO\%20DE\%202011.pdf>. Acessado em 08 de agosto de 2016.

FRUKI, Relatório sócio ambiental, 2014. Disponível em: <http://www.fruki.com.br/ upload/balancos/ATTITUDE_FOL_FRUKI_210x300_BALANCO_SOCIAL_2014_ LIBERfinalsite.pdf $>$. Acessado em 02 de setembro de 2016.

HELLER, Léo; DE PÁDUA, Valter Lúcio. Abastecimento de água para consumo humano. Editora UFMG, 2006.

HESPANHOL, Ivanildo. Reúso potável direto e o desafio dos poluentes emergentes. Revista USP, n. 106, p. 79-94, 2015.

LIU, Changhao; ZHANG, Kai; ZHANG, Jiaming. Sustainable utilization of regional water resources: experiences from the Hai Hua ecological industry pilot zone (HHEIPZ) project in China. Journal of Cleaner Production, v. 18, n. 5, p. 447-453, 2010.

MAPA, Ministério da Agricultura Pecuária e Abastecimento, 2000. Disponível em: <www2. agricultura.rs.gov.br/uploads/126989542129.03_enol_in_5_00_sda_mapa.doc>.Acesso em 02 de setembro de 2016.

MIERZWA, José Carlos; HESPANHOL, Ivanildo. Água na indústria: uso racional e reúso. Oficina de Textos, 2005. 
MORAIS, V. A. D. et al. - Avaliação microbiológica de amostras de refrigerantes comercializadas no Estado de Minas Gerais. Rev. Inst. Adolfo Lutz, 62(1): 1 - 4 ,2003

NUNES, José Alves. Tratamento físico-químico de águas residuárias industriais. Editora J. Andrade, 2004.

RIBEIRO, Wagner Costa. Aquífero Guarani: gestão compartilhada e soberania. Estudos avançados, v. 22, n. 64, p. 227-238, 2008.

RICHTER, Carlos A.; NETTO, José M. de Azevedo. Tratamento de Água, 1991.

RODRIGUES, Raquel dos Santos. As dimensões legais e institucionais do reuso de água no Brasil: proposta de regulamentação do reuso no Brasil. 2005. Tese de Doutorado. Universidade de São Paulo.

SEBRAE, Apreender a Empreender. Fundação Roberto Marinho, 2010. p. 147-158.

TCHOBANOGLOUS, George; BURTON, Franklin L.; STENSEL, H. D. Metcalf \& Eddy,(2003). Wastewater engineering: Treatment and reuse, v. 4.

TELLES, Dirceu D'Alkmin (Coord.); COSTA, Regina Helena Pacca Guimarães (Coord.).Reúso da água: conceitos, teorias e práticas. 2a Ed. São Paulo: Blucher. 2010. 408p. 